\title{
Identifier les attitudes des étudiants en géographie après leurs études secondaires
}

\author{
Burckin Dal (Istanbul Technical University)
}

\begin{abstract}
Résumé:
Ce travail expose les résultats d'une étude réalisée dans le but d'analyser la démarche d'apprentissage d'un groupe d'étudiants de premier cycle de géographie. Seront traitées ici les démarches d'apprentissage qu'ils adoptent et la façon dont évolue leur niveau de confiance en soi après un an d'enseignement supérieur. Les étudiants étaient confrontés à un programme visant au développement des capacités dans le cadre de la géographie, qui mettait l'accent sur une démarche d'apprentissage en profondeur. Les résultats montrent que, bien que leur niveau de confiance en leur capacité d'étudier et d'apprendre ait augmenté, leur démarche d'apprentissage est devenue de plus en plus instrumentale.
\end{abstract}

\begin{abstract}
:
This paper shows the results of a study carried out in order to analyse the learning approach in geography of cohorts of students on entry to a geography degree. After one year of higher education, student learning approaches and their degree of confidence are examined. A program aimed at the development of the learning capacities based on a deep learning approach was proposed to students. The results indicate that although their degrees of confidence in their capacity to study increased, their learning approaches became increasingly instrumental.
\end{abstract}

\section{INTRODUCTION}

Afin d'envisager la meilleure façon de concevoir des programmes scolaires qui développent chez les étudiants une capacité d'apprendre qu'ils garderont tout au long de leur vie, il faut tout d'abord que nous comprenions bien comment ils apprennent. Giordan et De Vecchi (1989), Chevallard (1991) et Astolfi (1995), notent qu'il ne sert à rien de modifier les programmes scolaires si l'on ne prend pas en compte les contextes d'apprentissage et si l'on ne se demande pas ce qu'apprendre signifie pour les étudiants. Des études ont permis d'identifier trois approches principales en matière d'apprentissage, approches que résume le Tableau 1. Ces approches sont couramment définies comme en profondeur, superficielle et instrumentale et stratégique (Marton et Saljo, 1976; Morgan et al., 1982; Astolfi et al., 1985).

Dans le contexte d'apprentissage, la qualité d'apprentissage de l'étudiant est déterminée par l'approche et la perception qu'a l'étudiant de l'apprentissage et elle est influencée par ses expériences d'apprentissage passées (Driver et al., 1985; Giordan 
et DeVecchi, 1987; Barth, 1987). De nombreuses études ont établi des liens très nets entre différents contextes d'apprentissage et la démarche d'apprentissage adoptée. Par exemple, l'apprentissage par problèmes est associé à une démarche de l'étudiant en profondeur et efficacité, en particulier à mesure qu'il progresse dans son parcours scolaire (Ramsden, 1987, Astolfi et Develay, 1989, Chevellard, 1991).

\begin{tabular}{|l|l|}
\hline Démarche en profondeur & Recherche du sens: intention de comprendre \\
& Lier les idées entre elles; lier les idées d'un cours à l'autre \\
Utiliser les preuves; lier les preuves aux conclusions \\
Aspects corrélatifs: \\
Intérêt pour les idées et les thèmes \\
Capacité de discuter et de collaborer
\end{tabular}

Tableau 1: Caractéristiques des démarches d'apprentissage (Marton et Saljo, 1976)

Les cours qui mettent fortement l'accent sur l'autonomie et peu sur les examens pour évaluer l'étudiant sont associés aux démarches en profondeur (Morgan et al., 1982 ; Astolfi et Develay, 1989 ; Retaillé, 1994). Au contraire, dans des institutions qui privilégient les examens, même s'il s'agit d'un apprentissage centré sur l'étudiant, les étudiants ont tendance à développer des démarches superficielles (Gilbert et al., 1982).

\section{Géographie et apprentissage de l'étudiant}

Les études de géographie sont considérées comme un processus qui prépare les étudiants à exercer une profession ou à faire des études de troisième cycle en leur donnant les capacités transmissibles qu'exigent les employeurs et les équipes de recherche (Audigier et al., 1996; Powell, 1997). Le secteur des études supérieures de géographie a également été proactif en reconnaissant l'évolution des objectifs dans l'éducation supérieure et l'importance croissante d'un apprentissage centré sur l'étudiant. C'est en partie pour répondre à ces évolutions qu'ont été développées 
des pratiques d'enseignement visant à former l'étudiant de sorte qu'il apprenne en profondeur plutôt que superficielle (voir tableau 1 pour une clarification des termes) (Astolfi et Develay, 1989; Hugonie, 1999).

On a cependant peu étudié l'attitude des étudiants de premier cycle de géographie en tant qu'apprenants ainsi que les démarches d'apprentissage qu'ils adoptent. Selon Brew et Boud (1995), les étudiants doivent se défaire des procédés d'apprentissage qu'ils ont utilisés pour passer le baccalauréat, basés sur des textes et des programmes fixes, et doivent développer la faculté critique nécessaire pour entreprendre des études universitaires. La démarche d'apprentissage de ces étudiants pouvait être qualifiée de superficielle ou instrumentale (Marton et Saljo, 1976; Morgan et al, 1982; Astolfi, 1985). Ils montrent une préférence pour les cours qui dispensent des informations et déclarent ne lire que pour les examens. Il est reconnu au sein de la communauté universitaire des géographes qu'afin de réussir leurs études de premier ou de deuxième cycle, les étudiants doivent former leur capacité d'apprentissage et adopter une démarche davantage en profondeur (Marton et Saljo, 1976; Ramsden et al., 1989; Maréchal et Genestier, 1992). La question pour les géographes est de savoir comment soutenir et opérer efficacement cette transition.

Cette étude est centrée sur un projet de programme scolaire qui a été introduit dans le but de développer les capacités des étudiants en géographie : capacités personnelles, capacités d'étudier et capacités transmissibles. En intégrant au sein du programme de géographie une formation visant à développer les capacités et en mettant l'accent de manière explicite sur une démarche « en profondeur », on espérait que les étudiants acquerraient de la maturité et se développeraient en tant qu'apprenants durant cette première année de transition.

\begin{tabular}{cccccc}
\hline $\begin{array}{l}\text { Âge } \\
\text { moyen }\end{array}$ & \% Baccalauréat & $\begin{array}{l}\text { Note moyenne } \\
\text { obtenue au } \\
\text { baccalauréat }\end{array}$ & $\begin{array}{l}\text { \% Entrée par des } \\
\text { voies non } \\
\text { traditionnelles }\end{array}$ & \% garçons & $\%$ filles \\
\hline 21 & 87 & $<12$ points & 13 & 40 & 60 \\
\hline
\end{tabular}

Tableau 2: Échantillon de statistiques démographiques utilisées dans cette étude ( $\mathrm{n}=145)$

\section{Méthodes}

Les étudiants choisis pour cette étude étaient en première année dans une petite université en Turquie. Tous commençaient un cursus de quatre ans d'études incluant plusieurs matières; la géographie constituait l'une de leurs trois matières durant la première année. Les données démographiques figurent dans le tableau 2.

Ces étudiants suivaient un programme de première année de géographie classique, consistant en cours magistraux, travaux dirigés (technologie de l'information, télédétection, cartographie et statistiques) et travail de terrain. De plus, ils assistaient à des séminaires de plusieurs heures chaque semaine, en petits groupes de 14-16 étudiants supervisés par un professeur. Le programme du séminaire avait été développé à partir d'un cours préexistant conçu par le projet, un fonds destiné au développement du projet d'enseignement et d'apprentissage [1], soutenu par le Ministère de l'Éducation en 2003. Le programme de ce séminaire visait à initier les 
étudiants aux études supérieures, en leur fournissant des occasions de développer des capacités transmissibles et interpersonnelles. En outre, en confrontant les étudiants à différentes expériences, on espérait renforcer leur confiance en soi dans ces domaines. Le programme couvrait les capacités et activités suivantes, adaptées aux études de géographie: travail de groupe; prise de notes; recherche de documents; recherches bibliographiques; écriture de dissertations; lecture critique, réflexion, analyse et discussion. La préférence revenait explicitement à l'apprentissage en profondeur, et les étudiants effectuaient différents exercices les aidant à mieux comprendre ce qu'est l'apprentissage en profondeur. Ceux-ci incluaient:

- lecture d'articles et prise de notes en adoptant une démarche d'apprentissage en profondeur puis superficielle afin de bien comprendre les différentes approches;

- évaluation d'échantillons de travaux appliquant des critères qui privilégient la compréhension des concepts géographiques et la capacité de les intégrer;

- évaluation critique et interprétation de données en géographie;

- discussion concernant des questions de géographie mettant en œuvre l'esprit critique;

- réflexion sur leur propre façon d'apprendre et sur leurs progrès.

Les étudiants étudiaient également deux autres matières et il serait inexact d'attribuer les changements observés au seul programme de géographie, car ces autres matières ont elles aussi contribué à développer la maturité des étudiants sur le plan universitaire.

L'étude comportait deux parties : une première étude des étudiants en première année de géographie à leur arrivée $(n=145)$, suivie d'une deuxième à la fin de l'année universitaire $(n=108)$. L'échantillonnage a eu lieu lors des sessions d'enseignement et le nombre d'étudiants présents déterminaient le nombre de questionnaires remplis. Ceux-ci avaient pour but d'identifier les tendances et les changements survenus dans la démarche d'apprentissage en géographie durant la première année d'études supérieures. Les résultats de cette étude seront analysés, afin d'identifier des moyens possibles de concevoir des programmes de géographie qui développent les capacités d'apprentissage des étudiants.

La première partie du questionnaire se composait des questions visant à identifier les caractéristiques démographiques et à évaluer le niveau de confiance en soi des étudiants dans des situations spécifiques qui surviendraient durant leur première année d'étude de la géographie (voir Figure 1, Annexe A). Plus précisément, il s'agissait d'évaluer la réussite du programme du séminaire portant sur les démarches d'apprentissage de l'étudiant, et d'analyser les faiblesses ainsi que les problèmes de confiance en soi.

Le second élément a été utilisé pour identifier les démarches d'apprentissage des étudiants. Le questionnaire mentionne trois degrés principaux (voir en Annexe B): en profondeur, stratégique et instrumental, instrumental incluant des approches superficielle et passives. Il traite aussi des raisons d'entreprendre des études universitaires, des éléments ayant une influence sur la capacité d'étudier et des préférences pour certains types d'apprentissage et de cours. 


\section{Étude préliminaire des démarches d'apprentissage des étudiants de premier cycle de géographie}

Les étudiants forment un ensemble très hétérogène, constitué d'individus ayant des styles d'apprentissage divers. Dans cette section, l'objectif était d'identifier les principales différences entre les démarches d'apprentissages adoptées par des sousgroupes de cet ensemble. Les données, une fois rassemblées, ont été classées selon des facteurs susceptibles de déterminer les différentes démarches d'apprentissage des étudiants. Des tests ont été réalisés sur les données pour déterminer les différences significatives existant entre les groupes de réponses. Il convient de noter que les échantillons fournis par certains sous-groupes sont de taille modeste et que de ce fait, les résultats les concernant doivent être traités avec prudence.

L'analyse de ces données montre qu'à l'évidence, ces étudiants de géographie présentaient des styles d'apprentissage variés, influencés par différents facteurs. Nombre de ces facteurs étaient prévisibles et avaient été identifiés auparavant, de façon anecdotique. Les étudiants ayant obtenu les meilleurs résultats au baccalauréat (plus de 15 points [2], à savoir 16 pour cent de l'échantillon, dont 44 pour cent de garçons, 56 pour cent de filles) montrent plus de confiance en leur capacité à étudier. Ces étudiants ne semblaient pas avoir le désir intrinsèque d'étudier la géographie, ce qui reflète peut-être la nature de ce cursus incluant plusieurs matières, où la géographie pouvait constituer leur deuxième, voire, pour certains d'entre eux, leur troisième matière. De plus, ils n'adoptaient pas une démarche d'apprentissage en profondeur, mais plutôt stratégique. Il pouvait s'agir d'une démarche qui s'était révélée efficace durant leurs études secondaires et qu'ils modifieraient au besoin au cours de leurs études supérieures, quand ils seraient confrontés à d'autres méthodes d'enseignement et d'évaluation (Chevellard, 1991; Audigier et al., 1996; Sharma, 1997). Chervel (1988) et Kember et Goow (1989) notent à partir de leurs études que les études secondaires privilégient la démarche superficielle et c'est ce que reflètent les démarches d'apprentissage des étudiants ayant obtenu des résultats médiocres au baccalauréat ( $\mathrm{n}=83$ pour cent) et des étudiants âgés de moins de 21 ans $(\mathrm{n}=88$ pour cent). Ces étudiants avaient une démarche instrumentale sans objectifs clairs et un manque de ligne directrice, associés à de nombreux facteurs indiquant des difficultés potentielles, susceptibles d'avoir un effet sur leurs études, par exemple le fait de pratiquer beaucoup d'activités sportives et de sortir souvent avec des amis, ainsi que des difficultés à étudier d'ordre général. Ils préféraient les cours durant lesquels les professeurs dictaient ce qu'il fallait noter et les examens pour lesquels il suffit d'apprendre ses notes. Comme on pouvait s'y attendre, ceux qui avaient obtenu les moins bons résultats avant d'entrer à l'université avaient une plus grande peur de l'échec et se sentaient plus déterminés à donner le meilleur d'eux-mêmes durant l'année que les étudiants ayant mieux réussi les examens d'entrée. Comment tirer parti de cette motivation, c'est ce que nous verrons par la suite.

Les étudiants entrés à l'université autrement que par la voie traditionnelle [3], dont 60 pour cent étaient plus âgés que la moyenne (plus de 22 ans) et qui avaient suivi des autres cours, tel que MYO, $(\mathrm{n}=13$ pour cent, 45 pour cent d'hommes, 55 pour cent de femmes) adoptaient une combinaison de démarches à la fois stratégiques et en profondeur, révélant une plus grande implication dans leurs études et un intérêt 
plus profond pour la matière étudiée. Les étudiants ayant suivi le programme MYO développent des démarches d'apprentissage interprétatives, à la fois imaginatives et prudentes, réfléchies et indépendantes. Ces étudiants révélaient une plus grande confiance en leur capacité d'aborder des situations susceptibles de se produire durant leur cursus, en particulier celles qui exigent des facultés interpersonnelles telles que poser des questions pendant un séminaire, faire des exposés et parler aux professeurs.

Les étudiants plus âgés, tous en outre entrés à l'université par des voies non traditionnelles ( $\mathrm{n}=11$ pour cent), semblent faire preuve d'implication dans leurs études et adopter une démarche stratégique par rapport à la gestion du temps, attitude leur permettant de tirer le meilleur parti de leurs études. Lorsque le temps le permet, les étudiants plus âgés saisissent les occasions qui se présentent de développer leurs propres idées et que, sur de nombreux points concernant leurs études, ils ont davantage confiance en eux que les étudiants plus jeunes. Il apparaît que les étudiants entrés à l'université par des voies non traditionnelles et les étudiants plus âgés ont une approche des études plus sérieuse et un intérêt plus profond pour les matières enseignées. Cela suggère que ceux-ci (du fait de leur formation et de leur expérience avant l'entrée à l'université, sur le plan scolaire et/ou social, et du fait qu'ils ont pris une décision réellement personnelle en choisissant de poursuivre leurs études), acquièrent différentes attitudes et démarches d'apprentissage qui s'avèrent préférables à celles des étudiants entrés par la voie classique, après le baccalauréat.

L'étude de Meyer (1995) et Severiens et Ten Dam (1998) qui recherchait des différences entre les démarches d'apprentissage déterminées par le sexe des individus, n'a pas donné de résultats probants. Les données fournies par cette étude révèlent que les femmes ( $\mathrm{n}=60$ pour cent) adoptent une démarche plus stratégique, et semblent organiser et gérer leurs études de façon plus efficace. Elle suggère qu'elles sont aussi plus susceptibles de collaborer avec d'autres personnes et craignent davantage l'échec que les hommes. Les résultats du questionnaire portant sur la confiance indiquent qu'à l'entrée à l'université, les étudiantes montrent moins de confiance en leurs capacités et aptitudes que les étudiants, fait dont nous commenterons plus tard les implications pour les professeurs de géographie. Toutefois, on note que les femmes étaient majoritaires parmi les étudiants plus âgés, qui manifestaient tous davantage de confiance en leurs capacités que les plus jeunes. Ceci souligne la diversité des approches au sein des groupes, diversité qui doit être prise en compte lors de l'élaboration des programmes scolaires.

\section{Évolution de l'apprentissage chez les étudiants de géographie}

Afin d'établir si ces programmes portant sur les capacités des étudiants avaient influencé leur démarche d'apprentissage, on a comparé, en utilisant des tests, les réponses données par les étudiants au début et à la fin de la première année universitaire au seconde élément du questionnaire et à celui qui évaluait leur niveau de confiance en soi. On trouvera plus bas un commentaire des résultats clés les plus significatifs au plan statistique.

\section{Les démarches d'apprentissage}

Les résultats montrent que les expériences faites la première année modifiaient les démarches d'apprentissage des étudiants, qu'elles diminuaient leur intérêt pour les 
idées et les concepts, leur signification et leurs relations. Ceci, accompagné d'une baisse d'intérêt pour les cours et un type d'enseignement basés sur une approche en profondeur, suggère l'adoption d'une démarche davantage instrumentale et superficielle. Selon les termes d'un étudiant, une démarche en profondeur est peutêtre plus intéressante mais demande plus de temps et en fin de compte, ce qu'il faut, c'est d'abord terminer tous les travaux demandés. Ces conclusions peuvent suggérer que, bien que le programme insiste sur une approche en profondeur, le message reçu par les étudiants est le suivant : la restitution telles quelles des informations lors des évaluations donne de bons résultats.

Les étudiants semblaient gagner en confiance à mesure que diminuait leur crainte de l'échec. Il y a un consensus sur le fait que l'anxiété nuit aux performances et par conséquent les étudiants moins anxieux sont plus à même de tirer le meilleur parti de leurs capacités. On s'interrogera plus loin sur les contradictions qui surviennent entre amélioration de la confiance en soi, absence d'une démarche en profondeur et réussite lors des évaluations.

Les étudiants manifestaient peu d'enthousiasme à l'égard de deux points qui sont caractéristiques d'une approche stratégique. Concernant le premier point, la maîtrise de l'efficacité, cela peut s'expliquer par l'importance assez excessive accordée à l'auto-évaluation en tant qu'élément formateur, introduite cette année-là. Des enquêtes réalisées auprès de groupes cibles par l'évaluateur externe du projet indiquaient que beaucoup d'étudiants tiraient peu profit de l'auto-évaluation et de l'auto-réflexion et les réduisaient à des tâches mécaniques et dépourvues de sens. Selon l'un des étudiants, il était facile d'écrire ce que les professeurs voulaient lire sans se donner beaucoup de mal ni réfléchir. Il est manifeste que les activités centrées sur l'auto-réflexion, introduites pour les aider à contrôler leur propre progression, n'avaient pas été intégrées au programme avec succès.

Il est difficile d'expliquer la faible motivation des étudiants à l'égard du deuxième point, réussir ; c'est peut-être parce que ces étudiants sont davantage capables de contextualiser la réussite ainsi qu'une conséquence directe de l'augmentation de leur confiance en soi. Il est possible qu'à ce stade de l'année universitaire, de nombreux étudiants aient déjà obtenu des notes suffisantes pour être sûrs de passer l'examen et que par conséquent ils ne soient pas très intéressés par le problème de la réussite dans le cadre d'un module réussir/échouer.

On observe un changement positif dans la démarche d'apprentissage des étudiants en ce qu'ils approuvent davantage un facteur que Giordan et De Vecchi, (1989) définissent comme incluant à la fois les capacités d'étudier habituelles et certaines des capacités transmissibles (par exemple la contribution à des discussions de groupe). Un résultat combiné concernant ceux-ci (utilisé dans le test) fournirait une indication générale de la confiance qu'ont les étudiants en leur capacité actuelle d'étudier. Ce qui signifie donc que les étudiants ont amélioré leur niveau de confiance en leur capacité d'apprendre et d'étudier à la fin du deuxième semestre, par rapport au premier. Grâce aux activités proposées par le programme du séminaire, ils ont appris les règles du jeu nécessaires pour suivre les cours et ne pas être noyé. 


\section{Niveaux de confiance}

Les réponses des étudiants aux questionnaire portant sur la confiance indiquent que le niveau de confiance en soi général du groupe avait augmenté au cours de l'année pour la plupart des points mentionnés, à l'exception de deux points, pour lesquels s'observait une diminution significative. Ces résultats soulignent les bénéfices tirés de ce programme visant à développer la confiance en soi dans les domaines qu'il couvrait. L'ordre respectif des réponses données au début et à la fin de l'année universitaire figure dans le tableau 3 et ces réponses sont commentées plus bas.

\begin{tabular}{|c|c|c|c|c|}
\hline Début du semestre 1 & $\begin{array}{l}\text { Résultat } \\
\text { donné par } \\
\text { rapport au } \\
\text { maximum } \\
\text { possible } \\
(\%)\end{array}$ & Fin du semestre 2 & $\begin{array}{l}\text { Résultat } \\
\text { donné par } \\
\text { rapport au } \\
\text { maximum } \\
\text { possible } \\
(\%)\end{array}$ & $\begin{array}{l}\text { Classement: } \\
\text { (Classement } \\
\text { précédent) } \\
1=\text { confiance } \\
\text { la plus faible }\end{array}$ \\
\hline Exposés individuels & 49 & $\begin{array}{l}\text { Temps pour les lectures } \\
\text { demandées }\end{array}$ & 57 & $1(5)$ \\
\hline S'en sortir financièrement & 55 & S'en sortir financièrement & 62 & $2(2)$ \\
\hline Gérer la quantité de & 58 & Exposés individuels & 63 & $3(1)$ \\
\hline travail & 60 & Rapport de groupe sur le & 63 & $4(15)$ \\
\hline $\begin{array}{l}\text { Ecrire la première } \\
\text { dissertation }\end{array}$ & 60 & $\begin{array}{l}\text { travail de terrain } \\
\text { Réaliser des affiches sur le } \\
\text { travail de terrain }\end{array}$ & 65 & $5(13)$ \\
\hline $\begin{array}{l}\text { Temps pour les lectures } \\
\text { au programme } \\
\text { Que faire en cas de } \\
\text { dépassement des délais }\end{array}$ & 63 & $\begin{array}{l}\text { Faire les bons choix pour la } \\
2^{\text {ème }} / 3^{\text {ème }} \text { année }\end{array}$ & 68 & $6(11)$ \\
\hline $\begin{array}{l}\text { Participer à des exposés } \\
\text { collectifs }\end{array}$ & 63 & $\begin{array}{l}\text { Que faire en cas de } \\
\text { dépassement des délais }\end{array}$ & & \\
\hline & 63 & Réussir tous les modules & 69 & $8(8)$ \\
\hline $\begin{array}{l}\text { Réussir tous les modules } \\
\text { Fournir un travail }\end{array}$ & 63 & & 69 & $9(3)$ \\
\hline $\begin{array}{l}\text { correspondant au niveau } \\
\text { exigé } \\
\text { Comprendre les cours }\end{array}$ & 64 & $\begin{array}{l}\text { Effectuer le travail de } \\
\text { terrain avec des personnes } \\
\text { que vous ne connaissez pas }\end{array}$ & 71.5 & $10(14)$ \\
\hline $\begin{array}{l}\text { Faire les bons choix pour } \\
\text { la } 2^{\text {ème }} / 3^{\text {ème }} \text { année }\end{array}$ & 66 & $\begin{array}{l}\text { Fournir un travail } \\
\text { correspondant au niveau }\end{array}$ & 72 & $11(9)$ \\
\hline $\begin{array}{l}\text { Poser des questions } \\
\text { pendant les séminaires }\end{array}$ & 66 & $\begin{array}{l}\text { exigé } \\
\text { Comprendre les cours }\end{array}$ & 72 & $12(10)$ \\
\hline $\begin{array}{l}\text { Réaliser des affiches sur } \\
\text { le travail de terrain }\end{array}$ & 69 & Écrire la première & 74 & $13(4)$ \\
\hline $\begin{array}{l}\text { Effectuer le travail } \\
\text { de terrain avec des }\end{array}$ & 69 & dissertation & 74 & $14(7)$ \\
\hline $\begin{array}{l}\text { personnes que vous ne } \\
\text { connaissez pas }\end{array}$ & 70 & $\begin{array}{l}\text { Participer à des exposés } \\
\text { collectifs }\end{array}$ & 74 & $15(16)$ \\
\hline travail de terrain & & $\begin{array}{l}\text { Avoir bien choisi les } \\
\text { matières à étudier }\end{array}$ & & \\
\hline
\end{tabular}




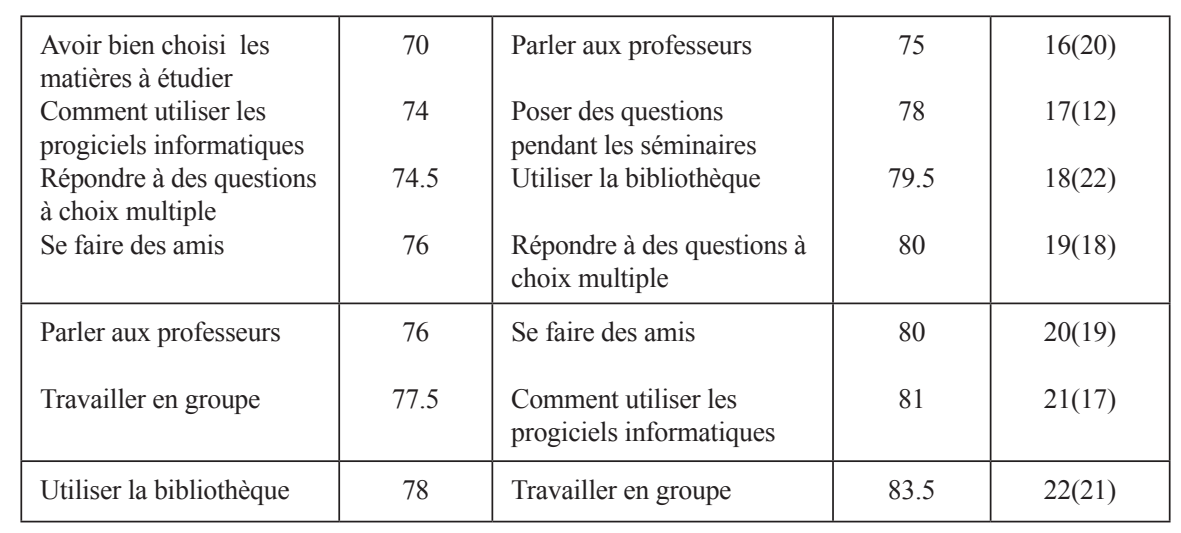

Tableau 3 : Niveaux de confiance des étudiants à accomplir diverses actions associées à l'étude universitaire au début et à la fin du semestre

Au début de leurs études universitaires, les préoccupations des étudiants semblaient tourner autour de problèmes d'intérêt immédiat tant sur le plan universitaire que personnel. Ils savaient que les premiers travaux qu'ils auraient à faire seraient une dissertation et un exposé collectif, travaux dans lesquels ils devraient montrer leur capacité de fournir un travail d'un niveau convenable. Les problèmes financiers préoccupaient à l'évidence de nombreux étudiants, qui devaient se débrouiller avec un budget d'étudiant pour la première fois. L'urgence de ces questions, ainsi que leur manque d'expérience pour les régler, apparaissent comme des facteurs affectant la confiance qu'ils avaient en leur capacité de s'en sortir. Les étudiants se disaient plus sûrs d'eux dans d'autres domaines, auxquels ils ne seraient confrontés que plusieurs mois plus tard, par exemple les rapports et les affiches relatifs au travail de terrain.

À ce stade, le point le plus préoccupant à leurs yeux était faire un exposé. L'exposé faisait déjà partie des travaux évalués lors de la première année, mais on l'avait remplacé par un exposé collectif pour ce groupe, dans l'idée que cela constituerait une introduction moins brusque à une activité qui intimidait les étudiants. Leurs réponses confirment cette opinion.

Il apparaît qu'en général la confiance en soi des étudiants a augmenté au cours de l'année. On a noté des progrès statistiques considérables portant sur de nombreuses activités et expériences incluses dans les séminaires (voir Figure 1, Annexe A) telles que : poser des questions pendant les séminaires ; exposés collectifs ; exposés individuels (pour une évaluation à visée formatrice) ; écrire la première dissertation ; fournir un travail correspondant au niveau exigé (critères d'évaluation couverts, remise d'un commentaire détaillé) ; gérer la quantité de travail (stratégies de gestion du temps couvertes) et comprendre les cours (prise de notes et stratégies de lecture efficace). L'aide apportée par le programme du séminaire ainsi que le fait d'avoir été confrontés à ces événements et d'y avoir fait face, ont dû contribué à augmenter la confiance des étudiants en leurs capacités. 
Il est intéressant d'examiner les points concernant lesquels les étudiants manifestaient une diminution de confiance significative, l'un de ces points étant le manque de temps pour faire toutes les lectures demandées. C'est une situation qui exige l'adoption d'une démarche d'apprentissage plus stratégique, mettant l'accent sur la gestion du temps et des stratégies de lecture efficace, afin de réussir.

$\mathrm{Au}$ début de l'année, les étudiants avaient confiance en leur capacité de parler aux professeurs. Cette confiance a considérablement diminué, et de façon préoccupante, au cours de l'année. Il se peut que le caractère chaleureux des expériences faites au début par les étudiants se soit un peu modifié à mesure qu'ils ont dû discuter avec les professeurs des questions de présence aux cours et de remise des travaux. Il est également possible que les professeurs, par manque de temps, n'aient pu se montrer aussi disponibles que prévu.

\section{Leçons tirées}

À partir des résultats de cette étude, il est possible d'identifier les besoins spécifiques de certains groupes d'étudiants ainsi que certaines faiblesses de ce programme portant sur les capacités, et de proposer des méthodes permettant de les corriger.

L'étude montre que les étudiants entrés à l'université avec des notes médiocres manifestent une tendance à être distraits par des sollicitations extérieures, malgré leur désir initial de réussir. Il faut donc détecter ce moment afin de les intéresser à la matière en question et de développer chez eux le sentiment qu'ils font partie du département dans lequel ils étudient. Pour y parvenir, deux méthodes complémentaires sont proposées. D'abord, il convient de s'assurer que les premiers éléments du programme de géographie sont actuels et qu'ils intéressent spécifiquement ces étudiants (on pourrait envisager en guise d'introduction une excursion sur le terrain, constituant une activité à la fois scolaire et sociale). Ensuite, les programmes pourraient être conçus de manière à permettre aux étudiants de réussir dès le début de l'année, par exemple grâce à une évaluation courte, simple dans sa forme (liée à l'excursion sur le terrain) qui serait notée et rendue rapidement à l'étudiant avec des conseils formateurs. Cette stratégie pourrait aussi s'appliquer à des étudiants ayant davantage de capacités mais manifestant un manque d'intérêt pour la matière en question.

Certaines étudiantes, en particulier les plus jeunes, se montrent moins sûres de leurs capacités et aptitudes. Une évaluation formatrice, effectuée tôt dans l'année, serait peut-être à même de résoudre ce problème, mais il faut également que les professeurs tiennent compte de ce point dans leur enseignement. S'assurer qu'elles ont autant de possibilités de contribuer aux discussions et maximiser les occasions de les encourager et de leur donner des conseils pourraient être des moyens efficaces. L'étude montrait que les étudiantes étaient davantage disposées au travail collectif, ce que l'on pourrait encourager en fournissant des occasions de travailler en groupe et en développant des groupes homogènes de soutien. Concernant le travail en petits groupes, il serait peut-être judicieux d'assurer un équilibre filles-garçons. Comme le notait une étudiante : J'étais la seule fille $d u$ groupe, avec un professeur homme, c'était difficile pour moi de prendre la parole.

Les étudiants plus âgés font preuve dans cette étude de créativité pour 
gérer leur temps efficacement. L'étude révèle qu'ils ont la faculté d'étudier en profondeur et de s'intéresser à la géographie. On pourrait cultiver ces qualités en leur permettant d'adopter des méthodes d'apprentissage moins traditionnelles. Mettre à leur disposition un matériel pédagogique orienté vers un apprentissage autonome (matériel papier et électronique) leur permettrait de concilier plus facilement l'apprentissage avec leurs autres responsabilités. De plus, en attribuant une place spécifique à des activités centrées sur l'étudiant dans leur emploi du temps, on valoriserait ce temps en question et on donnerait ainsi à ces étudiants la justification dont ils ont besoin pour se consacrer à ces activités.

Le projet de programme de géographie portant sur les capacités a atteint son objectif qui consistait à renforcer la confiance en soi des étudiants et à leur faire mieux prendre conscience de leurs capacités et aptitudes. Il n'a cependant pas atteint l'objectif consistant à encourager les étudiants à adopter une démarche d'apprentissage plus en profondeur. Les étudiants ont bien compris les différentes démarches d'apprentissage mais ont adopté une démarche de plus en plus instrumentale. Cela peut s'expliquer de deux manières. Tout d'abord, peut-être délivrons-nous des messages contradictoires la première année, en encourageant une approche en profondeur en géographie tout en récompensant les étudiants qui restituent correctement les informations que nous avons transmises. Ce fait risque d'être exacerbé par l'influence qu'exercent les deux autres matières de l'étudiant. C'est aussi ce que pensent Astolfi et Develay(1989) et Giordan et De Vecchi (1989) quand ils affirment que s'il s'avère nécessaire d'intervenir pour modifier les démarches d'apprentissage des étudiants, par exemple en introduisant une formation pour développer leurs capacités d'étudier, le changement espéré ne se produit pas forcément. Une étude réalisée auprès d'étudiants de Stanford University, aux ÉtatsUnis, a montré que la mise en place d'un programme portant sur les capacités d'apprentissage avait pour effet de modifier la démarche d'apprentissage des étudiants, qui passait de très superficielle (avant le test) à en profondeur et efficace (après le test) et d'améliorer considérablement les notes obtenues pendant l'année (Torrance, 1977). Une étude similaire menée à Melbourne University (Ramsden et al., 1986) débouchait sur des conclusions inverses puisque l'intervention sur les capacités d'apprentissage avait pour conséquence le renforcement de la démarche superficielle. La différence clé entre ces études paraît être les critères d'évaluation en vigueur pendant l'année dans chaque institution, la première exigeant des étudiants qu'ils réfléchissent pour réussir, tandis que la deuxième, selon la perception qu'en avaient les étudiants eux-mêmes, exigeait d'eux la restitution des informations. Dans les deux cas, les étudiants semblent conformer leur démarche d'apprentissage à la perception qu'ils ont des critères en vigueur dans leurs cours, même si cette perception est inexacte. Il s'avère que si nous voulons parvenir à développer chez les étudiants l'apprentissage en profondeur, nous devons compléter cette initiative en examinant soigneusement et holistiquement les stratégies d'évaluation de leur programme universitaire. Comment y parvenir dans des institutions où les étudiants choisissent la géographie comme une matière s'ajoutant à d'autres, exerçant elles aussi une influence, nous ne le savons pas avec certitude. De plus, les étudiants reconnaissent, semble-t-il, les avantages de cette démarche sur le plan intellectuel, 
mais, du fait des contraintes de la vie étudiante d'aujourd'hui, ils lui préfèrent une démarche plus instrumentale. Pour y remédier, on pourrait envisager des façons différentes de concevoir les cours et les évaluations, qui refléteraient l'évolution des besoins du corps étudiant sans nuire à la qualité du procédé d'apprentissage.

\section{CONCLUSION}

Sur la base de cette étude, on peut dire que lorsque les étudiants abordent leur cursus de géographie, ils sont en possession d'un mélange complexe de démarches d'apprentissage et que par conséquent les universitaires doivent prendre en compte l'hétérogénéité de leurs étudiants. Des suggestions ont été faites en vue de répondre aux besoins de ceux-ci. On observe dans les programmes de géographie une tendance à intégrer au sein du cursus des formations portant sur les capacités afin d'améliorer et de mettre en valeur les capacités et les aptitudes qu'ont les étudiants à apprendre. Cette étude a montré que les étudiants ayant suivi un cours de géographie spécifique, qui incluait un programme de séminaires visant à renforcer les capacités transmissibles et interpersonnelles, ont manifesté davantage de confiance en soi et modifié leur démarche d'apprentissage après leur première année universitaire. Suivre un programme orienté vers le développement des capacités a contribué à augmenter leur confiance en soi dans de nombreux domaines. Cependant, la tentative d'inciter les élèves à adopter vis-à-vis de leurs études une démarche plus en profondeur semble avoir échoué, du fait qu'elle n'était pas pleinement intégrée et soutenue dans l'ensemble du cursus de géographie et le reste du programme à ce niveau.

\section{Notes:}

${ }^{1}$ Cette initiative avait pour objectif de soutenir des projets visant à stimuler des évolutions dans le domaine de l'enseignement et de l'apprentissage et d'encourager la diffusion de pratiques valables dans le secteur de l'éducation supérieure en Turquie.

2 Les notes du baccalauréat dans le système turc sont des points accordés sur la base d'un système dans lequel les notes $\mathrm{A}, \mathrm{B}, \mathrm{C}, \mathrm{D}$ et $\mathrm{E}$ correspondent respectivement à 10, 8, 6, 4 et 2 points.

${ }^{3}$ En Turquie, il existe des programmes universitaires tel que MYO (pré-licence) qui durent 2 ou 3 ans après le baccalauréat traditionnel, et qui préparent les étudiants à exercer une profession. Après avoir terminé leur diplôme de pré-licence, les étudiants ont la possibilité de continuer les programmes de licence dans les universités. 


\section{Bibliographie:}

Astolfi J. P., 1985, Procédures d'apprentissage en sciences expérimentales, Paris, Inrp.

Astolfi J. P., 1995, Vers une pédagogie constructiviste, Lyon, Association Voies livres.

Astolfi J. P., Develay M., 1989, La didactique des sciences, Paris, Puf.

Audigier F., Cremieux C., Mousseau M.J., 1996, L'enseignement de l'histoire et de la géographie en trosième et seconde; étude descriptive et comparative, coll. Doc. Et travaux de recherche en éducation, Inrp.

Barth B., 1987, L'apprentissage de l'abstraction-methodes pour une meilleure réussite de l'école, Retz.

Brew A., Boud D., 1995, Research and learning in higher education, In : B. Smith \& S. Brown (Eds) research, Teaching and Learning in Higher Education, London, Kogan Page

Chervel A., 1988, L'histoire des disciplines scolaires, Histoire de l'Education, Inrp.

Chevallard Y., 1991, La transposition didactique, Grenoble, La Pensée Sauvage.

Driver R., Guesene E., Tiberghein, A., 1985, "Some features of children's ideas and their implications for teaching”, In R. Driver, E. Guesene, A. Tiberghin (eds) Children' ideas in science, Milton Keynes, Open Universty Press.

Gilbert J. K., Osborne, R.J., Fensham P.J., 1982, “Children's science and its consequences for teaching", Science Education, 66, 623-633.

Giordan A., De Vecchi G., 1989, L'enseignement scientifique : commet faire pour que ça marche? Nice, Z'éditions.

Hugonie G., 1992, Pratiquer la géographie au college, Paris, A.Colin.

Kember D., Gow L., 1989, "A model of student approaches to learning encompassing ways to influence and change approaches", Instructional Science, 18, 263-288.

Maréchal J., Genestier M., 1992, Le raisonnement géographique, dans l'enseigner la géographie du collège au lycée, Crdp d'Amiens.

Marton F., Saljo R., 1976, "On qualitative differences in learning, I: Outcome and process", British Journal of Educational Psychology, 46, 4-11.

Meyer J.H.F., 1995, "Gender group differences in the learning behaviour of entering first year students", Higher education, 29, 201-215.

Morgan A., Taylor E., Gibbs G., 1982, "Variations in students' approaches to studying", British Journal of Educational Technology, 13, 107-113.

Powell A., 1997, A Handbook of post sixteen geography, Sheffield, The Geographical Association.

Ramsden P., 1987, "Improving teaching and learning in higher education: the case for a relational perspective", Studies in Higher Education, 12, 275-286.

Ramsden P., Martin, E. Bowden, J., 1989, "School environment and sixth form pupils' approaches to learning", British Journal of Educational Psychology, 59,129-142.

Retaillé D., 1994, "Evaluer en géographie", L’information géographique, 5, 211-219.

Severiens S., Ten Dam G., 1998, "Gender and learning: comparing two theories", Higher education, 35,329-350.

Sharma, D.S., 1997, “Accounting students' learning conceptions, approaches to learning and the influence of the learning-teaching context on approaches to learning", Accounting Education, 6(2), 125-146.

Torrance, E.P., 1977, Creativity in the Classroom, Washington, DC, National Education Association. 
Annexes

Annexe A

Figure 1 : Les réponses des étudiants au questionnaire portant sur la confiance.
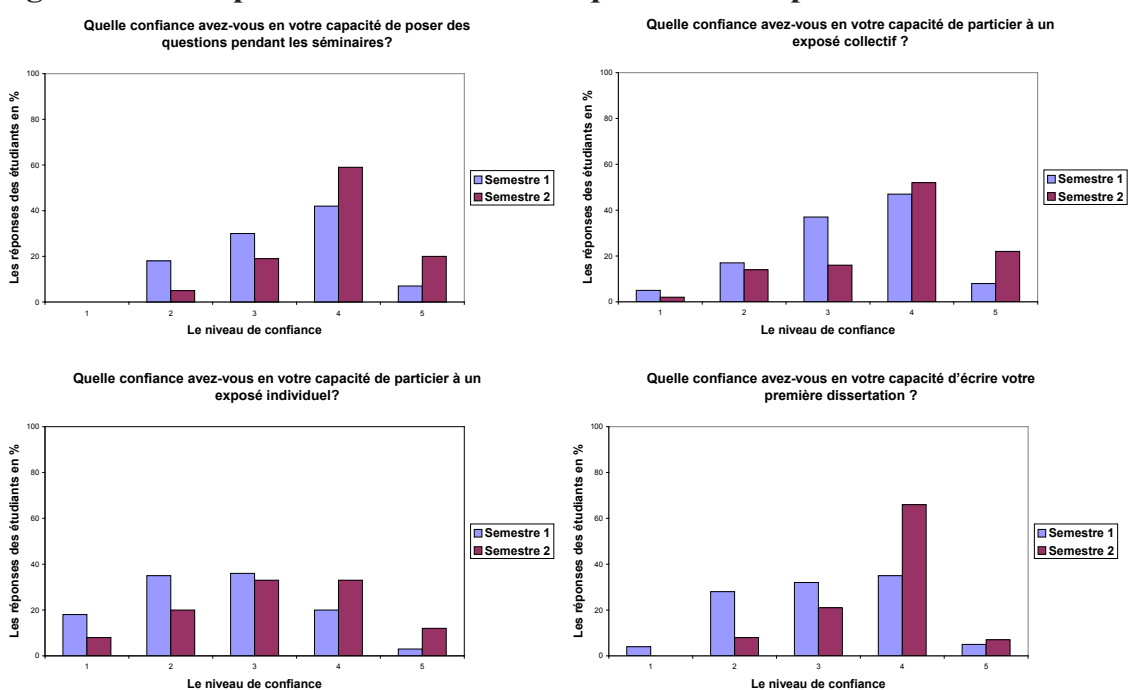

Quelle confiance avez-vous en votre capacité de comprendre les cours?
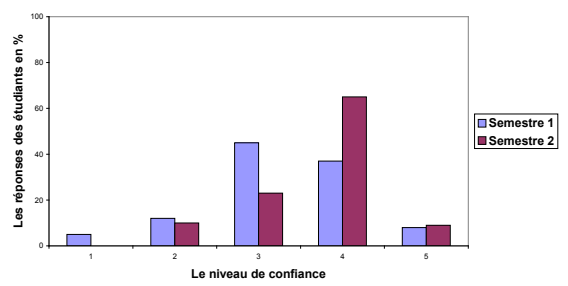

Quelle confiance avez-vous en votre capacité de fournir un travail correspondant au niveau exigé ?

Quelle confiance avez-vous en votre capacité de gérer la quantité de travail?
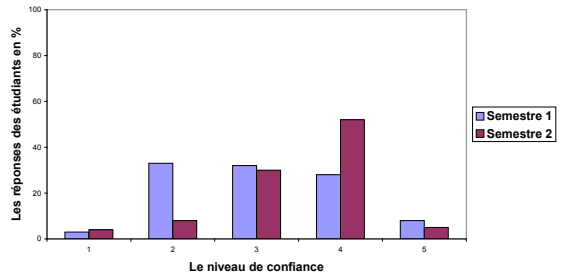

Quelle confiance avez-vous en votre capacité de trouver les temps nécessaire pour faire les lectures demandées ?

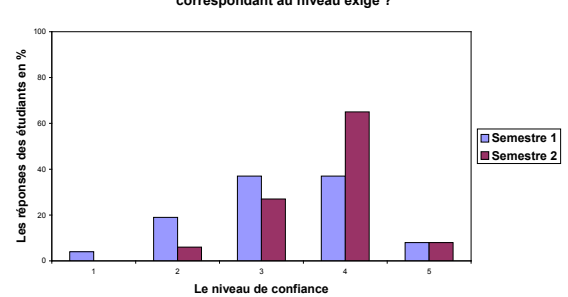

Quelle confiance avez-vous en votre capacité de vous en sortir financièrement ?

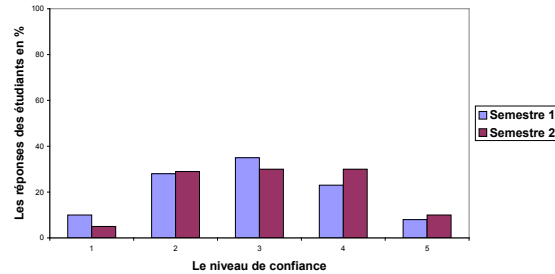

Quelle confiance avez-vous en votre capacité de parler avec les professeurs?
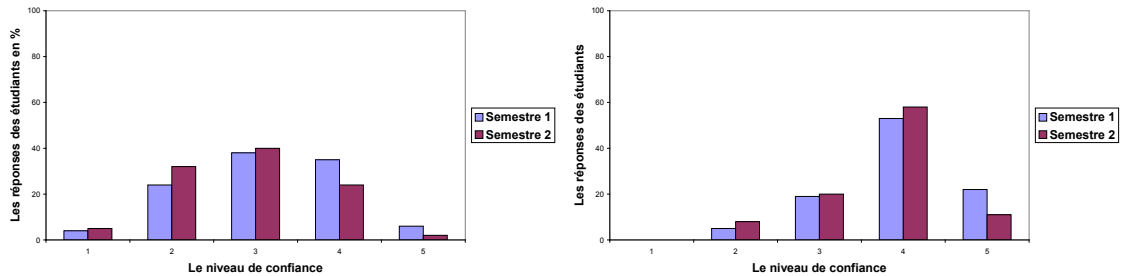

102 Canadian and International Education Vol. 38 no. 1 - June 2009 
4. J'essaie en général de comprendre l'intérêt pour moi-même de ce que nous devons apprendre. 54321

5. J'organise soigneusement mon temps de travail pour en faire le meilleur usage possible. 54321

6. J'ai l'impression de devoir surtout me concentrer sur la mémorisation d'une grande partie de ce que je dois apprendre. 54321

7. Je reviens attentivement sur le travail que j'ai fait pour vérifier que le raisonnement se tient. 54321

8. J'ai souvent l'impression d'être noyé dans le flot de données à traiter et à assimiler. 54321

9. J'examine attentivement ce qui a été démontré (les preuves) et j'essaie d'arriver à mes propres conclusions quant à ce que j'étudie. 54321

10. C'est important pour moi d'avoir l'impression que, dans ces cours, je donne vraiment le meilleur de mes capacités. 54321

11. J'essaie autant que possible de relier les idées que je rencontre avec celles qui me sont fournies par d'autres sujets ou d'autres cours. 54321

12. J'ai tendance à lire très peu en dehors de ce qui m'est demandé pour passer l'examen. 54321

13. Je me surprends souvent à réfléchir à des idées suscitées par les cours même quand je suis en train de faire autre chose. 54321

14. Je pense être assez systématique et organisé quand il s'agit de réviser pour les examens. 54321

15. J'examine attentivement les commentaires faits par les professeurs sur les travaux réalisés pour comprendre comment obtenir une meilleure note la prochaine fois. 54321

16. Le travail que je dois fournir ici me semble rarement intéressant ou pertinent sur le plan personnel. 54321

17. Quand je lis un article ou un livre, j'essaie de comprendre pour mon profit personnel ce que l'auteur veut dire exactement. 54321

18. J'ai la capacité de me mettre au travail dès que je dois le faire. 54321

19. Ce que j'étudie me semble souvent manquer de cohérence : ce sont des éléments sans lien les uns avec les autres. 54321

20. Je réfléchis à ce que je veux tirer des cours pour ne pas me disperser quand j'étudie. 54321

21. Quand je travaille sur un nouveau sujet, j'essaie de réfléchir personnellement à la façon dont toutes les idées s'articulent. 54321

22. J'ai souvent peur de ne pas arriver à faire le travail correctement. 54321

23. Il m'arrive souvent de remettre en question ce qui est dit pendant les cours ou ce que je lis dans des livres. 54321

24. J'ai l'impression d'avancer, ce qui m'encourage à m'investir davantage dans mon travail. 54321

25. Je me limite à apprendre les informations dont j'ai besoin pour passer les examens. 54321

26. Etudier des thèmes dans le cadre universitaire peut parfois être très stimulant. 54321

27. J'arrive bien à effectuer certaines lectures conseillées par les professeurs. 54321

28. J'ai présent à l'esprit le professeur qui va noter tel ou tel travail et les attentes qu'il est susceptible d' avoir. 54321

29. Quand je réfléchis, je me demande parfois pourquoi j'ai décidé de venir ici. 54321 
30. Quand je lis, je m'arrête de temps en temps pour réfléchir à ce que je compte tirer de ma lecture. 54321

31. Je travaille régulièrement durant le trimestre ou le semestre, plutôt que de tout faire à la dernière minute. 54321

32. Je ne suis pas vraiment sûr de savoir ce qui est important dans les cours, donc j'essaie de noter tout ce que je peux. 54321

33. Les idées que je rencontre dans des livres utilisés pendant les cours ou dans des articles suscitent souvent chez moi une longue réflexion personnelle. 54321

34. Avant de répondre à une question posée dans un devoir ou lors d'un examen, je m'interroge d'abord sur la meilleure façon de l'aborder. 54321

35. J'ai souvent tendance à paniquer quand je suis en retard dans mon travail. 54321

36. Quand je lis, j'examine attentivement les détails pour voir comment ils s'articulent à ce qui est dit. 54321

37. Je m'investis beaucoup dans mes études parce que je suis déterminé à réussir. 54321

38. Je me concentre uniquement sur ce qui semble être exigé dans les travaux à remettre et lors des examens. 54321

39. Je trouve passionnantes certaines idées que me fournissent les cours. 54321

40. Je planifie généralement à l'avance ma semaine de cours, soit par écrit soit dans ma tête. 54321

41. J'essaie de remarquer ce que les professeurs ont l'air de juger important pour me concentrer dessus. 54321

42. Je ne suis pas très intéressé par ces cours, mais je dois les suivre pour d'autres raisons. 54321

43. Avant de m'attaquer à une question ou un travail à remettre, j'essaie d'abord de comprendre quelles sont les implications. 54321

44. En général, j'utilise bien mon temps pendant la journée. 54321

45. J'ai souvent du mal à voir le sens des choses que je dois retenir. 54321

46. J'aime manier mes propres idées même si elles ne me mènent pas très loin. 54321

47. Quand j'ai terminé un travail, je le revois en détail pour voir s'il correspond bien à ce qui était demandé. 54321

48. Il m'arrive souvent de ne pas pouvoir dormir parce que j'ai peur de ne pas venir à bout d'un travail. 54321

49. C'est important pour moi de suivre l'argumentation, ou de trouver un fil directeur. 54321

50. Je ne manque pas du tout de motivation. 54321

51. J'aime qu'on me dise précisément ce que je dois faire dans le cadre des dissertations ou des travaux à remettre en général. 54321

52. Les sujets étudiés à l'université me passionnent parfois à tel point que j'ai envie de les approfondir. 54321

\section{Préférences pour différents types de cours et d'enseignement}

5 =j'aime beaucoup

$4=j$ 'aime assez

$2=$ je n'aime pas beaucoup

$1=$ je n'aime pas du tout

Essayez de ne pas utiliser 3, à moins que vous ne puissiez pas faire autrement ou que

l'affirmation ne puisse pas s'appliquer aux cours que vous suivez. 
a. les professeurs qui nous disent exactement ce qu'il faut noter. 54321

b. les professeurs qui nous encouragent à penser par nous-mêmes et nous montrent comment ils pensent eux-mêmes. 54321

c. les examens qui me permettent de montrer que j'ai réfléchi par moi-même à ce qui a été fait en cours. 54321

d. les examens et les évaluations pour lesquels il suffit d'utiliser nos notes de cours. 54321

e. les cours dans lesquels on nous dit très clairement quels livres il faut lire. 54321

f. les cours dans lesquels on nous encourage à faire beaucoup de lectures personnelles autour des sujets étudiés. 54321

g. les livres qui demandent un effort et fournissent des explications plus approfondies que les cours. 54321

h. les livres qui contiennent des informations et des faits précis que l'on peut apprendre facilement. 54321

Pour finir, comment pensez-vous avoir réussi les travaux remis jusqu'ici ?

Veuillez vous évaluer objectivement, en vous basant sur les notes que vous avez obtenues.

Très bien Assezbien Moyennement Pastrès bien Plutôt mal

$\begin{array}{lllllllll}9 & 8 & 7 & 6 & 5 & 4 & 3 & 2 & 1\end{array}$

\section{Méthode de notation}

Les étudiants répondent aux items en utilisant une échelle allant de 1 à 5 (5 étant le maximum). Les résultats des sous-échelles sont obtenus en additionnant les réponses apportées aux items sur ces sous-échelles. Les résultats concernant les trois démarches principales sont obtenus en additionnant les résultats des sous-échelles qui contribuent à déterminer chaque démarche. On peut calculer ces résultats en utilisant un ordinateur, grâce à un programme. Chaque item est considéré comme une variable (par exemple D04 = item en profondeur 4), ensuite un total sur la sous-échelle est obtenu en créant une nouvelle variable qui est la somme des items. Par exemple, Recherche du sens $(\mathrm{RS})=\mathrm{D} 04+\mathrm{D} 17+\mathrm{D} 30+\mathrm{D} 43$. Ensuite, les démarches peuvent être crées de la même manière Démarche en profondeur $(\mathrm{DP})=\mathrm{RS}+\mathrm{RI}+\mathrm{UP}+\mathrm{II}$.

Démarche en profondeur

Recherche du sens

$4-17-30-43$

\section{Relier les idées}

$11-21-33-46$

\section{Utilisation des preuves}

$9-23-36-49$

Intérêt pour les idées (sous-échelle correspondante)

$13-26-39-52$

Démarche stratégique

Etude de manière organisée

$1-14-27-40$

Gestion du temps

$15-18-31-44$ 
Prise en compte des critères d'évaluation

$2-15-28-41$

Détermination à réussir (sous-échelle correspondante)

$10-24-37-50$

Gestion de l'efficacité (sous-échelle correspondante)

$7-20-34-47$

Démarche superficielle et passive

Manque de ligne directrice

$3-16-29-42$

Mémorisation mécanique

$6-19-32-45$

Limitation au programme

$12-25-38-51$

Peur de l'échec (sous-échelle correspondante)

$8-22-35-48$

Préférences pour différents types de cours et d'enseignement Résultat formé par la somme de quatre items.

Qui aide à comprendre (liée à une démarche en profondeur)

$\mathrm{b}-\mathrm{c}-\mathrm{f}-\mathrm{g}$

Qui transmet des informations (liée à une démarche superficielle)

$\mathrm{a}-\mathrm{d}-\mathrm{e}-\mathrm{h}$

Dr. Burckin Dal teaches in the Department of Humanities and Social Sciences at Istanbul Technical University and can be reached at dalbu@itu.edu.tr. 